\title{
Monodisperse Branched Molybdenum-Based Bioactive Nanoparticles Significantly Promote Osteogenic Differentiation of Adipose-Derived Stem Cells
}

\author{
Wen Niu, Yi Guo, Yumeng Xue, Mi Chen, Min Wang, Wei Cheng, and Bo Lei**
}

\begin{abstract}
Adipose-derived stem cells (ADSCs) are considered to be ideal stem cell sources for bone-tissue regeneration owing to their ease of collection and high activity. However, the regulation of osteogenic differentiation of ADSCs using biomaterials without adding growth factors is still not satisfactory. For the first time, molybdenum-doped bioactive glass nanoparticles with a radial porous morphology (Mo-rBGNs) are reported and their role in the osteogenic differentiation of ADSCs is investigated. The results show that Mo-rBCNs exhibit radially porous and spherical morphology, relatively homogeneous particle size $(200-400 \mathrm{~nm})$, and excellent apatiteforming bioactivity. They do not affect the proliferation of ADSCs, but significantly regulate their osteogenic differentiation and biomineralization. 5\% Mo-rBGNs significantly enhance the alkaline phosphatase activity and biomineralization ability and promote the osteogenic gene expressions of collagen I secretion and bone sialo protein in ADSCs. A reasonable and promising strategy for designing nanoscale bioactive materials with the excellent osteogenic ability for stem cell-based bone tissue regeneration is provided.
\end{abstract}

\section{Introduction}

Due to the growing trauma, disease, and aging of population, bone tissue defect has been a relatively common clinical disease. ${ }^{[1]}$ There was increasing requirement for biomaterials to replace/repair/restore the bone tissue. ${ }^{[2,3]}$ In the field of bone tissue regeneration, stem cell-based therapy has been recognized as a promising therapeutic option, due to their versatile differentiation capacity and bioactivity. ${ }^{[4,5]}$ As a kind of multipotent cells, mesenchymal stem cells (MSCs) retain their potential of self-renewal and can differentiate into multiple kinds of cell types under suitable microenvironments. ${ }^{[6]}$ As a type of mesenchymal stem cells, bone marrow MSC (BMSC) is considered to be an ideal candidate for bone tissue regeneration. ${ }^{[7-9]}$ However, in recent years, more attention has been given to the promotion of osteogenesis in adipose-derived stem cells (ADSCs). ${ }^{[10]}$ As compared to BMSCs, ADSCs have numerous advantages, including abundant sources, ease of isolation, and a higher propensity to be accepted by patient. ${ }^{[11]}$ The regulation of the osteogenic differentiation of ADSCs is rather essential for their clinical applications in the bone tissue regenerative medicine. ${ }^{[12,13]}$

Various bioactive factors such as miRNA and protein/peptide have been employed to treat diseases and enhance the osteogenic differentiation of BMSCs/ADSCs. ${ }^{[14-17]}$ However, the bioactive factors usually show several disadvantages including high cost and low stability. ${ }^{[18]}$ Relative to conventional bioactive factors, recently bioactive materials, which can efficiently
W. Niu, Y. Guo, Y. Xue, M. Chen, M. Wang, W. Cheng, Prof. B. Lei Frontier Institute of Science and Technology

Xi'an Jiaotong University

Xi'an 710054, China

E-mail: rayboo@xjtu.edu.cn

Y. Guo

Department of Materials Science and Engineering

University of Michigan

Ann Arbor, MI 48109, USA

Y. Xue

Department of Bioengineering

Department of Chemical and Biomolecular Engineering

Henry Samueli School of Engineering and Applied Sciences

University of California-Los Angeles

Los Angeles, CA 90095, USA

The ORCID identification number(s) for the author(s) of this article can be found under https://doi.org/10.1002/ppsc.201900105.
Prof. B. Lei

Instrument Analysis Center

Xi'an Jiaotong University

Xi'an 710054, China

Prof. B. Lei

Key Laboratory of Shaanxi Province for Craniofacial Precision Medicine

Research

College of Stomatology

Xi'an Jiaotong University

Xi'an 710000, China

DOI: 10.1002/ppsc.201900105 
regulate the cell behavior and tissue regeneration, have been developed through their optimized design for the structurecomposition. ${ }^{[19-22]}$ The bioactive materials were also developed for improving the osteogenic differentiation of stem cells. ${ }^{[23-25]}$ For example, bioactive silicate-based biomaterials and bioactive glasses (BGs) have shown their inherent osteogenic differentiation ability for both BMSCs and ADSCs. ${ }^{[26,27]}$ BGs have become one of the best bioactive materials for bone regeneration in recent years, due to their biocompatibility, osteoinduced ability, and controlled degradation, as well as bone-bonding activity. ${ }^{[28-34]}$ Recent studies showed that bioactive materials with nanostructure demonstrated the enhanced capacity for enhancing soft/hard tissue regeneration in vitro and in vivo. ${ }^{35-38]}$ It was very necessary to develop novel nanoscale bioactive materials for stimulating the osteogenic differentiation of ADSCs.

In previous studies, our group developed several monodispersed bioactive glass nanoparticles (BGNs) for biomedical applications including bioimaging, gene/drug delivery, and osteogenic differentiation of stem cells. ${ }^{[39-42]}$ For example, monodispersed BGNs possessed inherent enhanced osteogenic differentiation ability for BMSCs and osteoblasts in vitro, as well as reinforced bone tissue regeneration in vivo. ${ }^{[43]}$ Our previous study first showed that monodispersed BGNs could enhance the osteogenic differentiation of ADSCs through activating the TGF-Beta/Smad 3 signaling pathway. ${ }^{[44]}$ In addition, the metallic elements, such as $\mathrm{Cu}, \mathrm{Sr}$, and $\mathrm{Gd}$, showed their special biological functions including stimulation of osteogenesis and angiogenesis, as well as antitumor activity. ${ }^{[45-47]}$ Previous study found that europium-doped BGNs (Eu-BGNs) significantly enhanced the osteogenic differentiation of human BMSCs. ${ }^{[48]}$ However, europium is not the essential element of human body and probably difficult to be cleared in renal, which has increased the concerns regarding the long-term toxicity. The effect of metallic elements-doped BGNs on the osteogenic differentiation of ADSCs is few reported.

By contrast, molybdenum is known to be readily absorbed from the intestinal tract and excreted mainly in the urine. ${ }^{[49]}$ Molybdenum is also very important for the normal metabolism of life as a necessary trace element of organisms. ${ }^{[50]}$ Furthermore, recent studies showed that molybdenum may be related to the growth of bone and tooth. ${ }^{[51]}$ Therefore, it may be very promising to investigate the molybdenum-doped BGNs (Mo-BGNs) as a bioactive material for regulating osteogenic differentiation of ADSCs.

In this study, we synthesize the branched monodispersed Mo-rBGNs with a radial porous structure and investigate their potential application in regulating the osteogenic differentiation of ADSCs. The physicochemical structure, biomineralization activity, cytotoxicity, as well as the effects on cellular mineralization, alkaline phosphatase activity (ALP), osteogenic protein, and gene expressions of ADSCs for Mo-rBGNs, were investigated.

\section{Results and Discussion}

\subsection{Synthesis and Characterizations of Mo-rBGNs}

In this work, to demonstrate the osteogenic differentiation effect of functionalized monodispersed BGNs, Mo-doped radial bioactive glass nanoparticles (Mo-rBGNs) were developed by a modified sol-gel method (Figure 1). The osteogenic activity of Mo-rBGNs was evaluated by detecting its effect on regulating the cellular biomineralization and expressions of osteogenic gene in ADSCs.

The physicochemical structure characterizations of Mo-rBGNs samples are shown in Figure 2. The as-prepared Mo-rBGNs exhibited a radially spherical morphology and a uniform diameter of 300-400 nm (Figure 2a-h; Figure S1, Supporting Information). As shown in transmission electron microscope (TEM) images (Figure 2e-h), the Mo incorporation did not produce effect on the size of Mo-BGNs, but the morphology was significantly tight upon the increase of Mo content, indicating the increase of Mo-complexes in glass network. The mean hydrodynamic diameters of 0Mo-rBGNs, 2Mo-rBGNs, 5Mo-rBGNs, and 10Mo-BGNs were about 308,

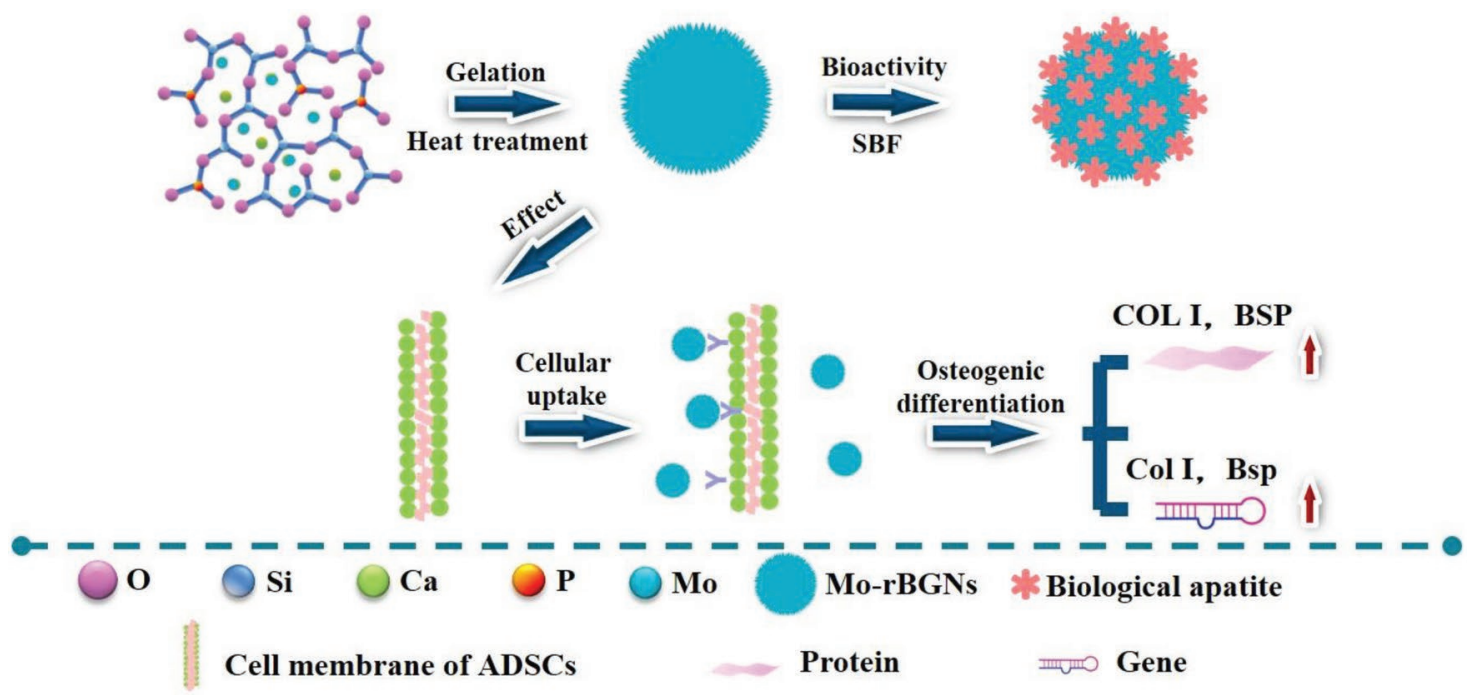

Figure 1. Schematic illustration describing the synthetic route of Mo-rBGNs and their potential biomedical applications in vitro and in vivo. 

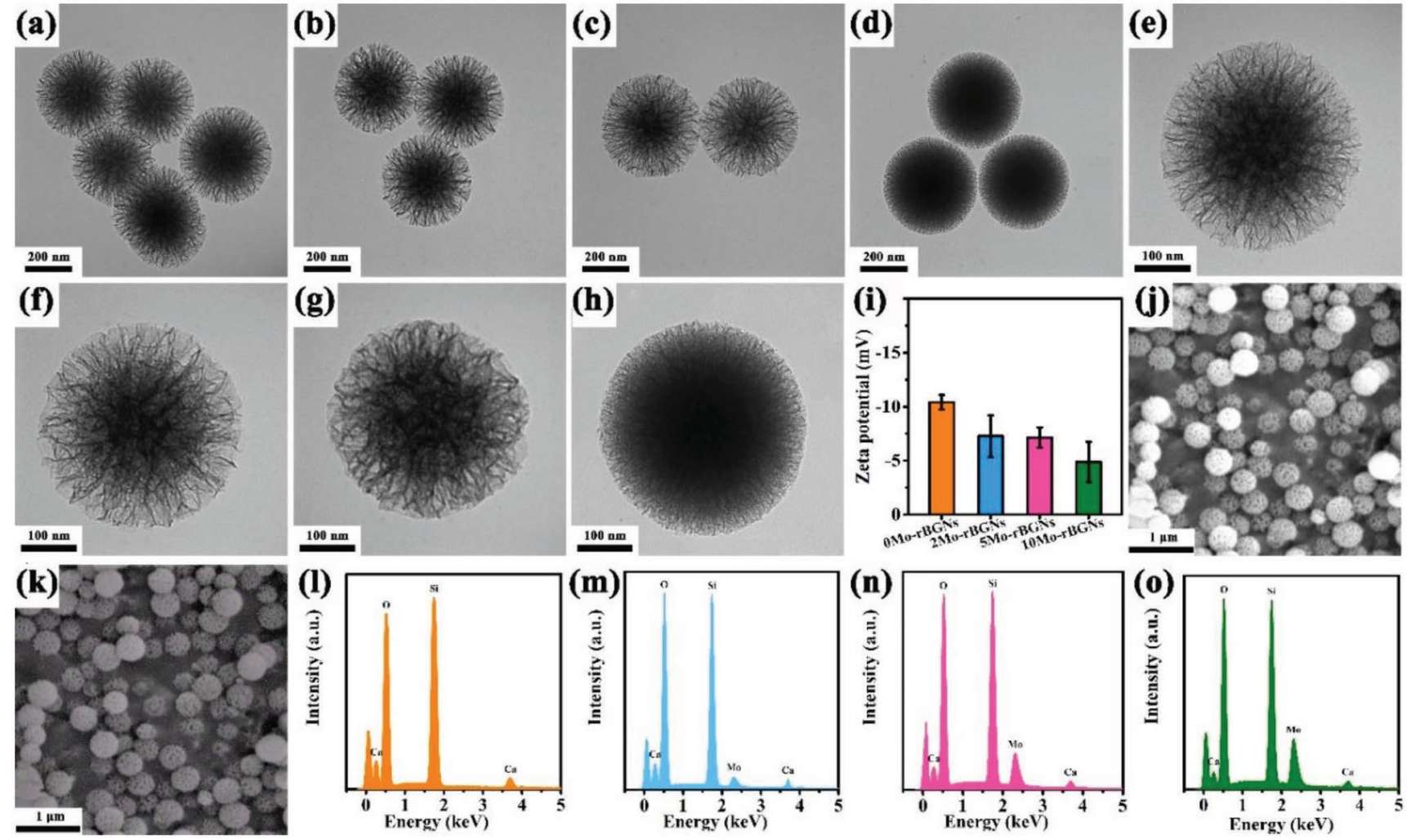

Figure 2. Morphology evaluation of Mo-rBGNs. a-d) TEM images of OMo-rBGNs a), 2Mo-rBGNs b), 5Mo-rBGNs c), and 10Mo-rBGNs d) (scale bar: $200 \mathrm{~nm}$ ); e-h) high magnification TEM pictures (scale bar: $100 \mathrm{~nm}$ ); i) Zeta-potential analysis (*p<0.05; $* * k<0.01$ ); j) SEM image of 10Mo-rBGNs (scale bar: $1 \mu \mathrm{m}$ ); k) EDS mapping of $10 \mathrm{Mo}-\mathrm{rBGNs}$ (scale bar: $1 \mu \mathrm{m}$ ); I-o) EDS spectra of OMo-rBGNs, 2Mo-rBGNs, 5Mo-rBGNs, and 10Mo-rBGNs.

330,320 , and $423 \mathrm{~nm}$ with Zeta potentials of $-10.4,-7.26,-7.13$, and $-4.89 \mathrm{mV}$, respectively (Figure $2 \mathrm{i}$ ). The morphology and element composition of Mo-rBGNs were also analyzed by high resolution scanning electron microscopy (SEM) (Figure 2j,k). The energy dispersive spectra (EDS) mapping of 10Mo-rBGNs showed that $\mathrm{Si}$ and Mo were evenly distributed within the whole nanoparticles (Figure S2, Supporting Information). The EDS analysis confirmed that the detected $\mathrm{Mo} / \mathrm{Si}$ molar ratio resulted in fair agreement with the theoretical ratio (Figure 2l-o). Additionally, as the increase of the intensity of Mo peak, the calcium (Ca) decreased because Ca was substituted by Mo complexes in the glass compositions. Such findings indicate that Mo was incorporated in the silicate structure of the glasses.

The phase structure and chemical structure of $x$ Mo-BGNs were further analyzed by X-ray diffraction (XRD), Fourier-transform infrared spectroscopy (FTIR), and X-ray photoelectron spectroscopy (XPS) (Figure 3). In the XRD patterns of Mo-rBGNs (Figure 3a), the broad peak at $23^{\circ}$ was corresponded to amorphous silica, and the diffraction peaks at $2 \theta=18.6^{\circ}, 28.8^{\circ}, 31.2^{\circ}, 34.3^{\circ}, 47.1^{\circ}, 49.3^{\circ}$, and $58.0^{\circ}$ were attributed to (101), (112), (004), (200), (204), (220), and (312) reflections of $\mathrm{CaMoO}_{4}$ (JCPDS 29-0351). The above analyses suggest that the phase structure of Mo-rBGNs was significantly affected by the addition of Mo. As shown in FTIR (Figure 3b), the bands located at around 860, 898, and $1000 \mathrm{~cm}^{-1}$ were assigned to the bending vibration of $\mathrm{Si}-\mathrm{O}-\mathrm{Si}$, the asymmetrical stretching mode of $\mathrm{Mo}-\mathrm{O}-\mathrm{Mo}$, and the symmetrical stretching mode $\mathrm{Si}-\mathrm{O}-\mathrm{Si}$, respectively.
Moreover, the presence of a band at around $1631 \mathrm{~cm}^{-1}$ was caused by the $-\mathrm{OH}$ groups in water molecules. The characteristic bands of $\mathrm{Si}-\mathrm{O}-\mathrm{Si}$ were significantly weakened upon the increase of Mo content, indicating the decrease of glass network integrity and the appearance of molybdenum phase. The XPS analysis showed that surface of 10Mo-BGNs was composed of $\mathrm{Ca}, \mathrm{O}, \mathrm{Si}$, and Mo elements (Figure 3c) and the typical XPS twopeak shape of Mo 3d spectrum could be observed (Figure 3d). This pair of strong peaks located at 231.2 and $234.5 \mathrm{eV}$ could be attributed to the $\mathrm{Mo}^{6+}$. These results demonstrated that monodispersed Mo-rBGNs with uniform size was synthesized successfully by the ultrasonic assisted template technique.

\subsection{Biomineralization Activity Analysis of Mo-rBGNs}

In order to explain the bone-bonding ability and osteogenic bioactivity of Mo-rBGNs, the samples were soaked into simulated body fluids (SBF) for various times to evaluate the biological apatite-forming ability (biomineralization activity). After immersion in SBF for $1 \mathrm{~d}$, the structure and morphology of Mo-rBGNs were not significantly changed (Figure 4a-c; Figure S3, Supporting Information). After further soaking in SBF, the XRD diffraction patterns (Figure 4d) of Mo-rBGNs demonstrated peaks at $\approx 31.7^{\circ}, 36.4^{\circ}, 45.4^{\circ}$, and $56.6^{\circ}$ which corresponded to the lattice planes (112), (202), (222), and (211) of hydroxyapatite (HA) (JCPDS 09-0432). And two characteristic peaks at 596 and $604 \mathrm{~cm}^{-1}$ corresponding to the $\mathrm{P}-\mathrm{O}$ bands in $\mathrm{PO}_{4}{ }^{3-}$ 


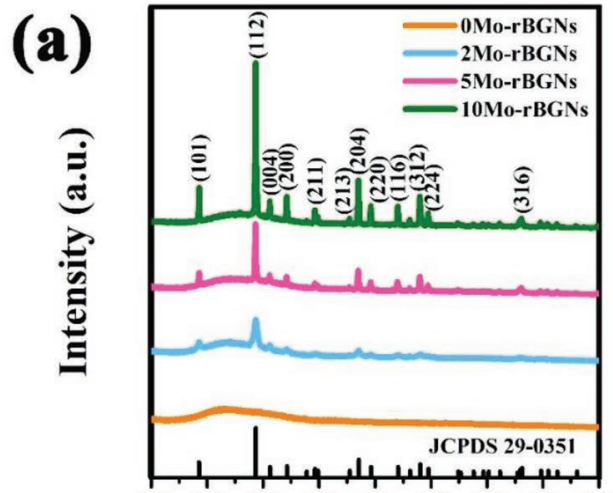

102030405060708090

$2 \theta$ (degree)

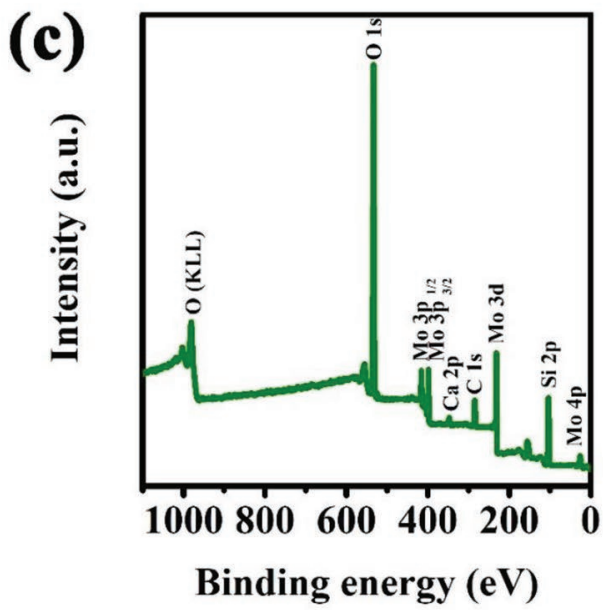

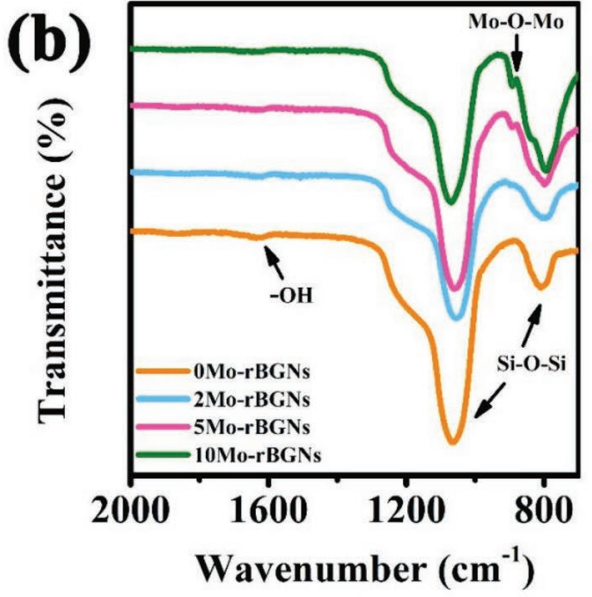

(d)

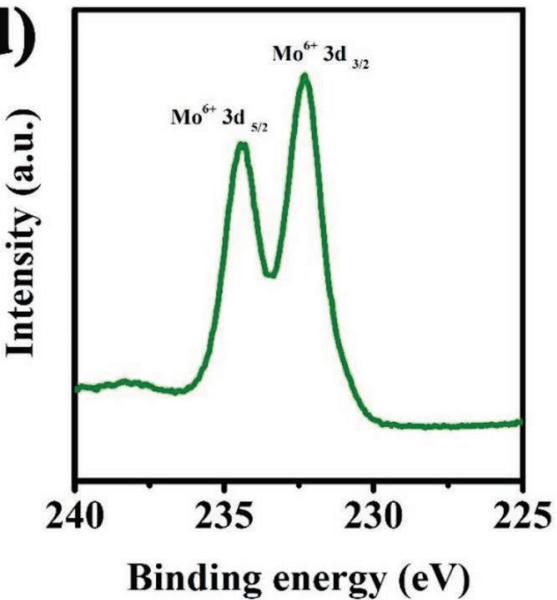

Figure 3. Characterizations of Mo-rBGNs. a,b) XRD patterns a) and FTIR spectra b) of Mo-rBGNs; c) XPS spectrum of 10 Mo-rBGNs; d) Mo $3 d$ peaks in XPS spectra of 10Mo-rBGNs.

of crystalline $\mathrm{Ca}-\mathrm{P}$ were seen in the FTIR spectra (Figure 4e) after soaking for $3 \mathrm{~d}$. These new peaks of FTIR spectra and XRD patterns became significantly stronger upon the increase of the soaking time (Figure $4 \mathrm{~g}, \mathrm{~h}$ ). Additionally, after soaking MorBGNs in SBF for 3 and $7 \mathrm{~d}$, TEM demonstrated that the deposited apatite layer possessed a flake-like morphology which was the typical morphology of hydroxyapatite (Figure 4f,i; Figures S4 and S5, Supporting Information). In summary, the above results confirmed the apatite-forming ability of Mo-rBGNs implying their possible bone-bonding capability in vivo and Mo incorporation did not affect the excellent biomineralization of BGNs.

\subsection{Cellular Biocompatibility Evaluation}

ADSCs were employed as the model to analyze the cellular biocompatibility of Mo-rBGNs. After incubation with samples (40-200 $\mu \mathrm{g} \mathrm{mL} \mathrm{m}^{-1}$ ), ADSCs exhibited good cell attachment morphology (Figure 5a; Figure S6, Supporting Information). Specifically, as shown in Figure 5a, after treatment with nanoparticles at $80 \mu \mathrm{g} \mathrm{mL} \mathrm{m}^{-1}$ for $72 \mathrm{~h}$, significantly higher numbers of living cells (green fluorescence) were observed as compared with Blank, demonstrating that samples at this safe concentration could support cell growth. However, at the high particle concentration (120 and $200 \mu \mathrm{g} \mathrm{mL}{ }^{-1}$ ), poor attachment morphology was observed between different Mo-rBGNs groups and Blank. These results indicated that the suitable concentration of Mo-rBGNs could improve cell proliferation. In addition, at day 1 , Mo-rBGNs with $40 \mu \mathrm{g} \mathrm{mL} \mathrm{m}^{-1}$ demonstrated significantly higher cell viability compared to that of Blank. The cell viability showed a significant decrease upon the increase of concentration (Figure 5b). Notably, the relative cell viability values of 5Mo-rBGNs showed that the cells could efficiently proliferate after culture for $72 \mathrm{~h}$ with nanoparticles at a concentration of $40-80 \mu \mathrm{g} \mathrm{mL}^{-1}$ (Figure 5c). Our results demonstrated that Mo-rBGNs with specific Mo content at a safe concentration could efficiently enhance cellular biocompatibility which may be a great benefit to their application of osteogenic differentiation.

\subsection{Osteogenic Differentiation Analysis}

The ALP activity and mineralized nodule formation are an earlystage marker for osteogenic differentiation and a phenotypic marker for mature osteoblasts. The ALP activities measured in ADSCs following incubation with Mo-rBGNs (30-90 $\left.\mu \mathrm{g} \mathrm{mL}^{-1}\right)$ 


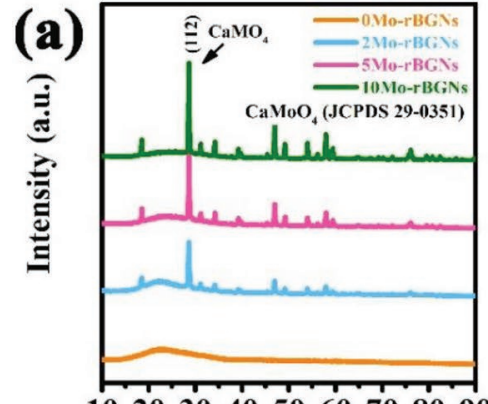

102030405060708090

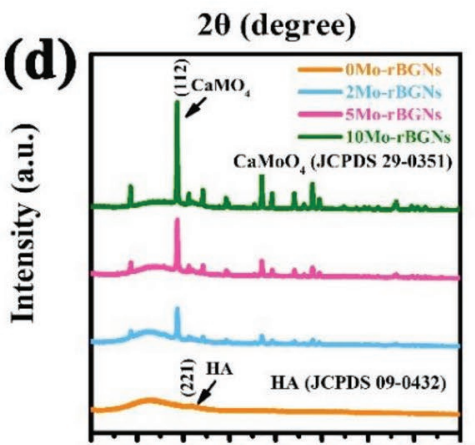

$\begin{array}{llllllllll}10 & 20 & 30 & 40 & 50 & 60 & 70 & 80 & 90\end{array}$

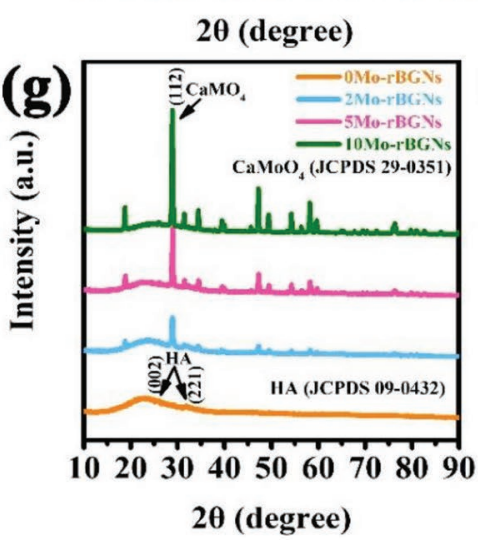

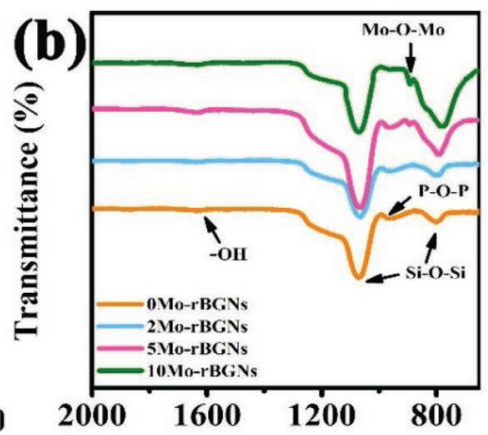
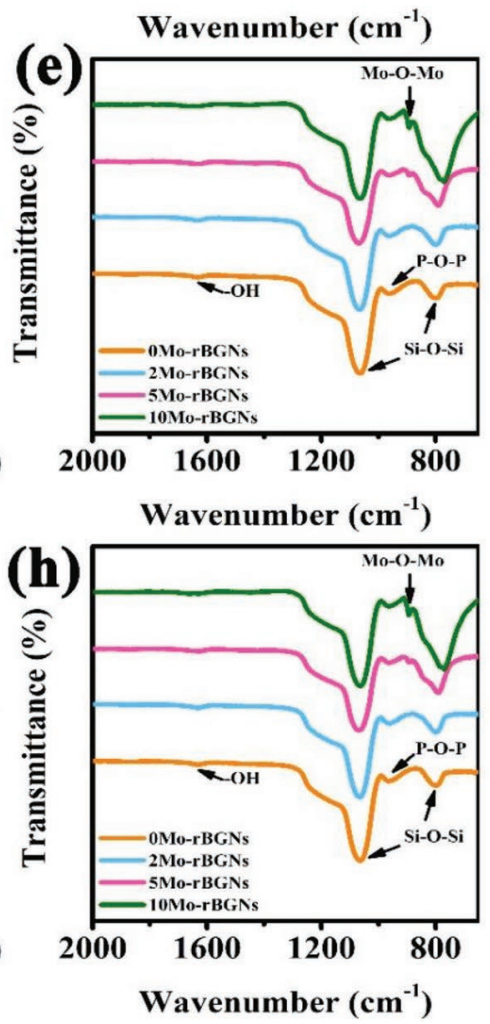

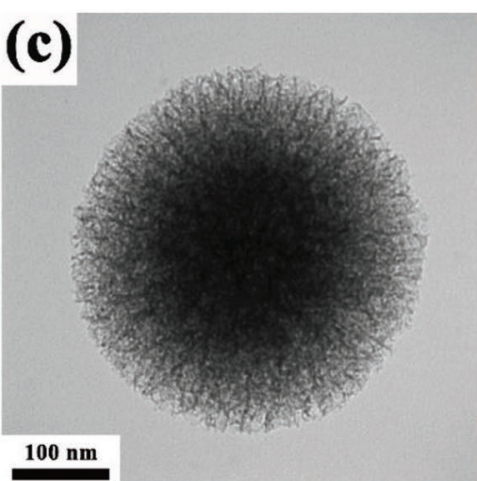

(f)
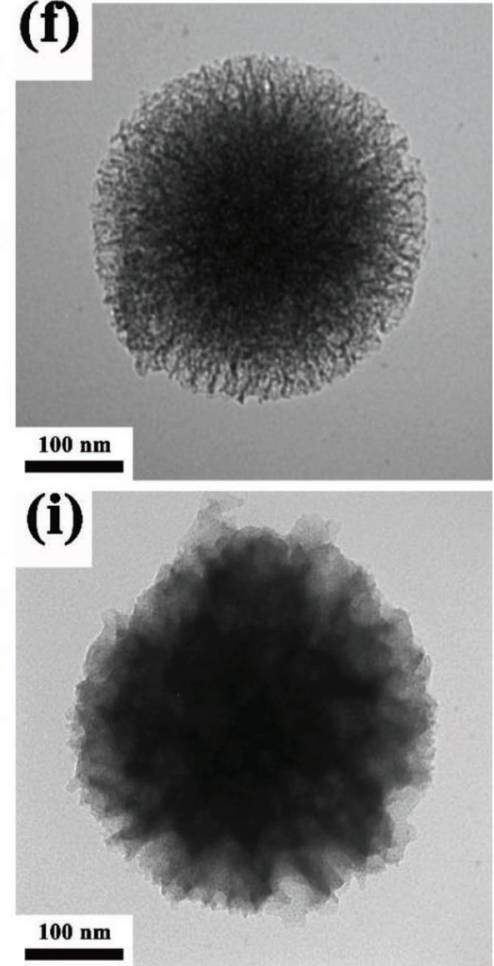

Figure 4. Biomineralization activity evaluation of Mo-rBGNs. a-c) XRD patterns a), FTIR spectra b) of Mo-rBGNs, and TEM image c) of 10Mo-rBGNs after biomineralization for $1 \mathrm{~d}$ (scale bar: $100 \mathrm{~nm}$ ); $d-f$ ) XRD patterns d), FTIR spectra e) of Mo-rBGNs, and TEM image $f$ ) of $10 M o-r B G N s$ after biomineralization for $3 \mathrm{~d}$ (scale bar: $100 \mathrm{~nm}$ ); g-i) XRD patterns g), FTIR spectra h) of Mo-rBGNs, and TEM image i) of 10Mo-rBGNs after biomineralization for $7 \mathrm{~d}$ (scale bar: $100 \mathrm{~nm}$ ).

under osteoinductive conditions were detected at days 7 and 14. Figure 6a,b shows that the relative ALP activities in all samples were significantly increased with culture times. On day 7 , for 2Mo-rBGNs and 5Mo-rBGNs, the ALP activities were significantly increased with increasing particle concentration, with no significant differences observed between other groups. After incubation with Mo-rBGNs from $30-90 \mu \mathrm{g} \mathrm{mL}^{-1}$ for $14 \mathrm{~d}$, the ALP activities were all higher than Blank. These results showed that low-content Mo (2 and 5 mol\%)-doped rBGNs with suitable concentration could effectively enhance the ALP activity of ADSCs. The calcium deposition and mineralization of Mo-rBGNs were detected by alizarin red stain and relative intensity calcium-content examination (Figure 6c,d). Compared with Blank, Mo-rBGNs groups with concentration of $60 \mu \mathrm{g} \mathrm{mL}$ demonstrated significantly stronger alizarin red staining after a $21 \mathrm{~d}$ incubation, suggesting positive calcium mineralization (Figure 6c). The relative gray intensity of calcium-content detection in ADSCs was performed following treatment with different concentrations of materials for $21 \mathrm{~d}$ (Figure 6d). We observed that the relative gray intensity of calcium content was significantly enhanced by incubation with 5Mo-rBGNs of $60 \mu \mathrm{g} \mathrm{mL}{ }^{-1}$ relative to the Blank; however, there were no significant difference in calcium content between the different concentration groups on day 21 (Figure 6d; Figure S2, Supporting Information). The results from alizarin red staining and relative gray intensity of calcium-content analysis suggested that the optimized Mo-doped rBGNs significantly improved mineralization capability of ADSCs.

To demonstrate the effect of Mo-rBGNs at the concentration of $60 \mu \mathrm{g} \mathrm{mL}-1$ on the osteogenic differentiation of ADSCs at 

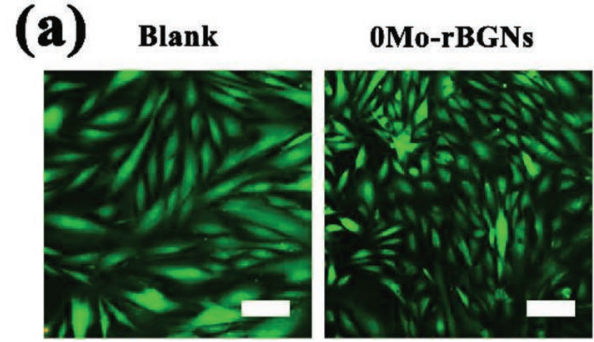

(b)

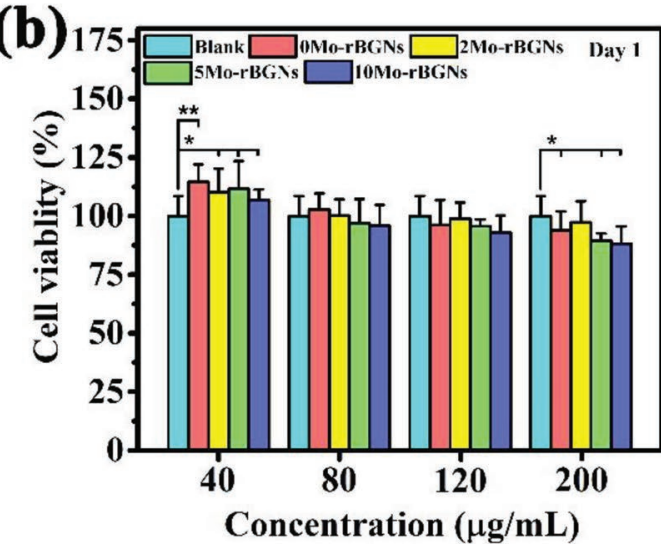

2Mo-rBGNs

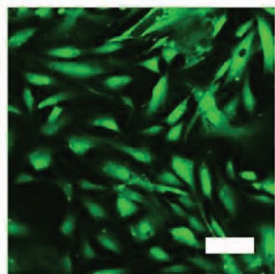

5Mo-rBGNs

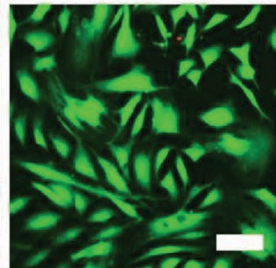

10Mo-rBGNs

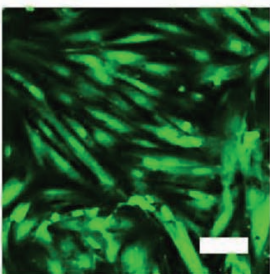

(c)

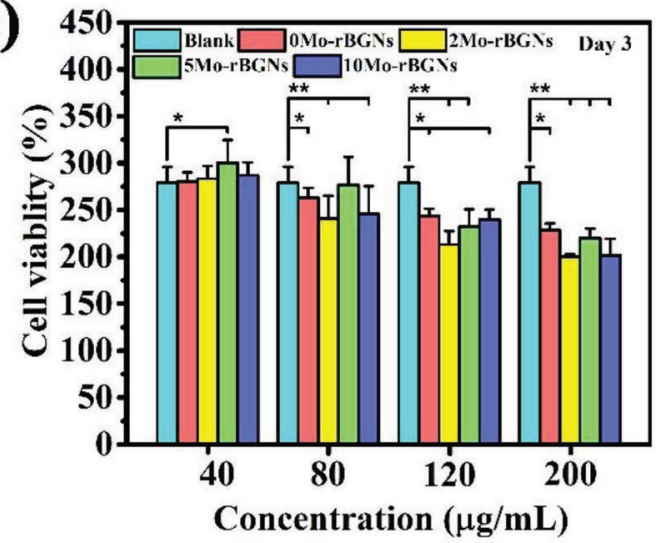

Figure 5. Cellular biocompatibility evaluation of ADSCs following incubation with various concentrations of Mo-rBGNs. a) Fluorescence images indicating live-dead staining after a $3 \mathrm{~d}$ incubation (scale bar: $100 \mu \mathrm{m}$ ); b,c) cell viability and proliferation after culture for $1 \mathrm{~d} \mathrm{~b}$ ) to $3 \mathrm{~d}$ c) (*p $<0.05$; $* * p<0.01)$. The solution without nanoparticle was used as the negative control (Blank).

the mRNA level, we determined the expressions of early-stage osteoblastic marker genes (Col I) and late-stage marker (Bsp) in cells after culture for various periods (Figure 7). The Col I expression on day 7 was first upregulated after incubation with Mo-BGNs at a low-content Mo-doped and followed by the decreased at 5Mo-rBGNs (Figure 7a). On day 14, no significant difference of Col I expression among various groups was observed (Figure 7b). For Bsp expression, on days 7 and (a)

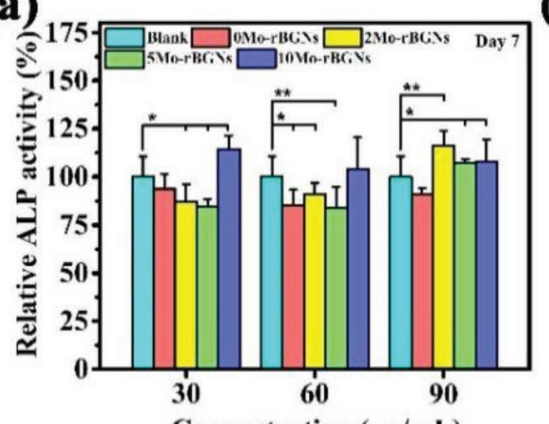

(c)

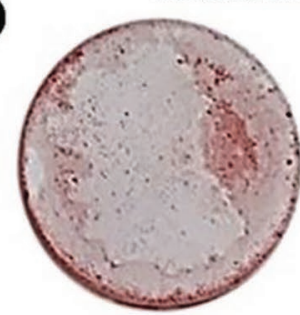

Blank (b)

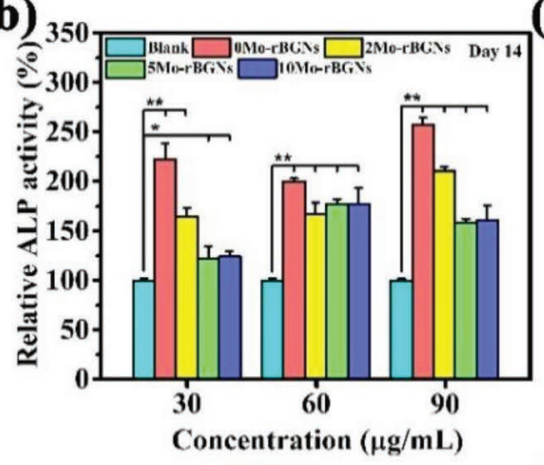

(d)

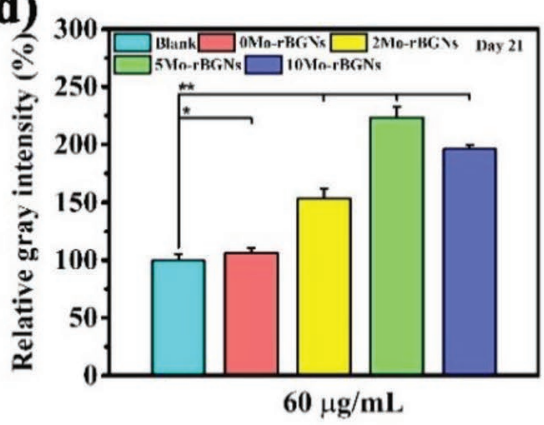

$n(\mu \mathrm{g} / \mathrm{mL})$

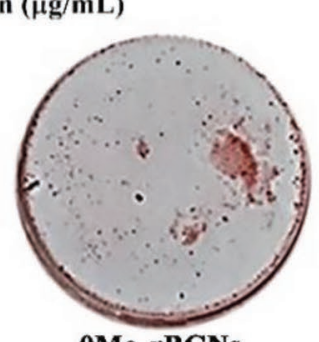

OMo-rBGNs

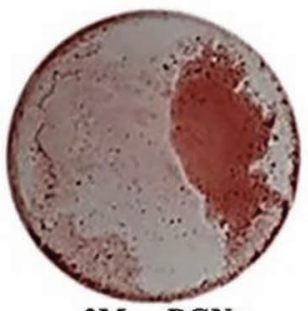

2Mo-rBGNs

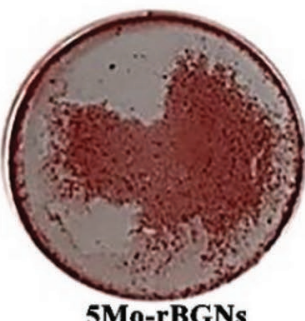

5Mo-rBGNs

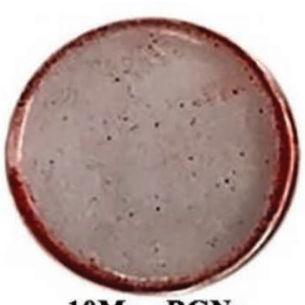

10Mo-rBGNs

Figure 6. In vitro ALP activity evaluation and calcium deposition biomineralization staining evaluation of ADSCs following induction by Mo-rBGNs. a,b) ALP activity on day 7 a) and day 14 b) (*p<0.05; **p<0.01); c) the relative gray intensity based on the images of alizarin red staining; d) alizarin red staining of ADSCs (*p<0.05; **p $<0.01)$. 
(a)

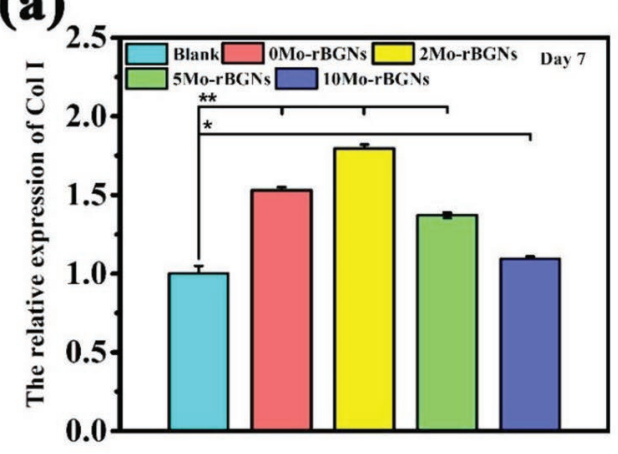

(c)

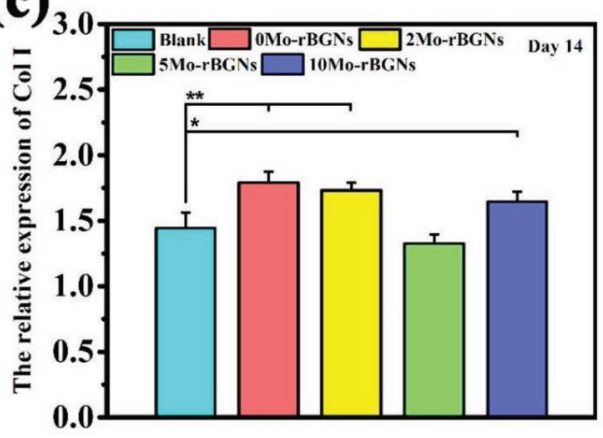

(b)

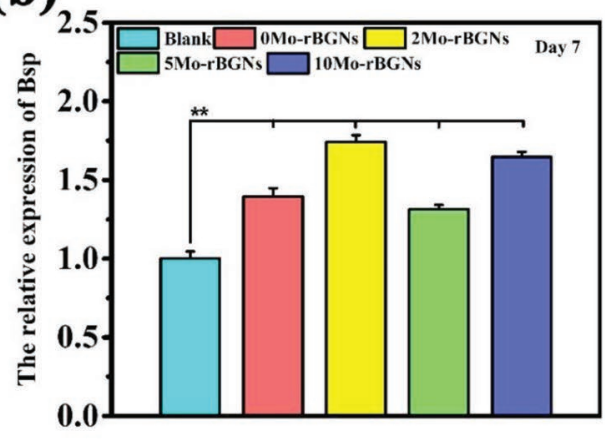

(d)

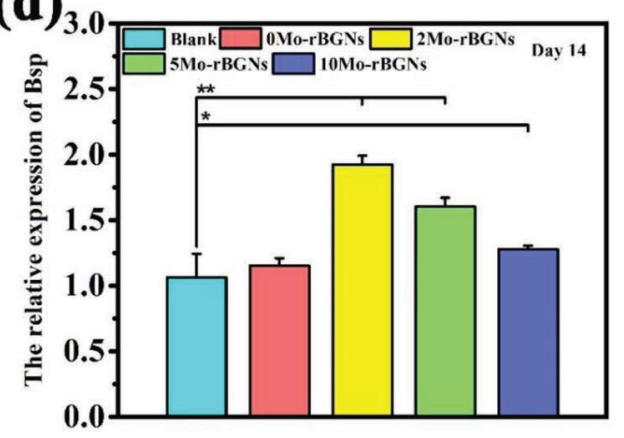

Figure 7. Relative expression of osteoblastic marker gene during osteogenic differentiation following induction by Mo-rBGNs with $60 \mu \mathrm{g} \mathrm{mL}^{-1}$ on day 7 and 14. a,b) Relative expression of Col I a) and Bsp b) on day 7; c,d) relative expression of Col I c) and Bsp d) on day 14. *p $<0.05$; ** $p<0.01$.

14, 2Mo-rBGNs group with $60 \mu \mathrm{g} \mathrm{mL}^{-1}$ showed the significantly high Bsp level, as compared with Blank and other groups (Figure 7c,d; Figure S7, Supporting Information). These results suggested that the Mo-rBGNs could dramatically enhance the osteogenic genes expression in ADSCs.

Additionally, the immunofluorescence staining for Col I and Bsp proteins on day 21 were investigated to demonstrate the continuity effect of Mo-rBGNs on ADSCs osteogenic proteins expression. Col I and Bsp were shown, respectively, as green and red while nuclei were stained as blue. Both of early- and late-stage proteins in Blank groups expressed no obvious positive Col I and Bsp staining (Figure 8a). However, the significant fluorescence intensity associated with Col I expression continued to increase from 0 to $5 \mathrm{~mol} \%$ of Mo-doped $\mathrm{rBGNs}$, reaching peak values at $5 \mathrm{~mol} \%$ (Figure $8 \mathrm{~b}$ ). Under the same osteoinductive condition, the Bsp protein expression showed a similar tendency with the positive Col I protein staining and 5Mo-rBGNs showed observably higher fluorescence intensity associated with Bsp expression comparing with other groups (Figure 8c). The ALP activity, alizarin red staining, osteogenic genes analysis, and immunofluorescence staining further demonstrated that Mo-rBGNs could efficiently enhance the osteogenic differentiation of ADSCs.

\section{Conclusion}

In summary, monodispersed Mo-rBGNs were fabricated by an ultrasonic assisted template method. Mo-rBGNs showed a uniform spherical porous morphology with a particle size of
300-400 nm. The Mo-rBGNs could efficiently induce the formation of biological apatite layer under SBF environment, indicating their excellent biomineralization activity. Our Mo-rBGNs significantly enhanced the osteogenic differentiation of ADSCs through improving their ALP activity, calcium mineralization, osteogenic proteins, and gene expressions. This study first suggests that the rBGNs with low content Mo doping could effectively enhance the biomineralization and osteogenic differentiation of ADSCs, suggesting their great potential for ADSCbased bone tissue regeneration.

\section{Experimental Section}

Synthesis of Monodispersed Mo-rBGNs: Monodispersed Mo-rBGNs was prepared by incorporating Mo into the BGNs framework to replace role of calcium through an ultrasonic assisted sol-gel method. Briefly, $7.5 \mathrm{~mL}$ cyclohexane was added into a solution composed of $0.15 \mathrm{~g}$ urea, $0.23 \mathrm{~g}$ isopropanol, $0.25 \mathrm{~g}$ cetylpyridinium bromide, and $7.5 \mathrm{~mL}$ of deionized water under continuous stirring at room temperature. The mixture was allowed to react for $2 \mathrm{~h}$ prior to the addition of $0.675 \mathrm{~mL}$ tetraethyl orthosilicate (TEOS). Under vigorous stirring of $30 \mathrm{~min}$, the reactants were heated to $70^{\circ} \mathrm{C}$ and were allowed to react for $7 \mathrm{~h}$. Then appropriate amounts of series of reagents were added in the following sequence: TEP, $\mathrm{Ca}\left(\mathrm{NO}_{3}\right)_{2} \cdot 4 \mathrm{H}_{2} \mathrm{O}, \mathrm{H}_{8} \mathrm{MoN}_{2} \mathrm{O}_{4}$, allowing $30 \mathrm{~min}$ for each reagent to react completely. After a further stirring of $16 \mathrm{~h}$, the suspension was centrifuged at $10000 \mathrm{rpm}$ for $15 \mathrm{~min}$ to collect deposits, which were further washed thrice with acetone, twice with ethanol, and twice with water. Finally, these deposits were dried by a freeze-drying before calcination at $650{ }^{\circ} \mathrm{C}$ for $10 \mathrm{~h}$ at a heating rate of $1{ }^{\circ} \mathrm{C} \mathrm{min}-1$ under air conditions. The designed compositions of Mo-rBGNs were $60 \mathrm{SiO}_{2}:(36-x) \mathrm{CaO}: 4 \mathrm{P}_{2} \mathrm{O}_{5}: x \mathrm{Mo}$ in $\mathrm{mol} \%(x=0,2$, 5 , or 10) and designated as 0Mo-rBGNs, 2 Mo-rBGNs, 5Mo-rBGNs, 
(a)

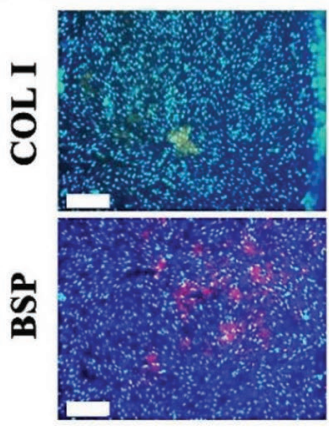

(b)

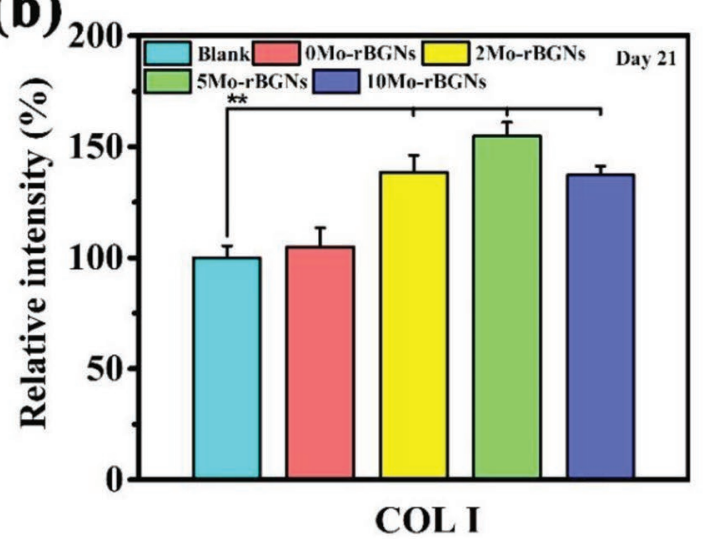

0Mo-rBGNs
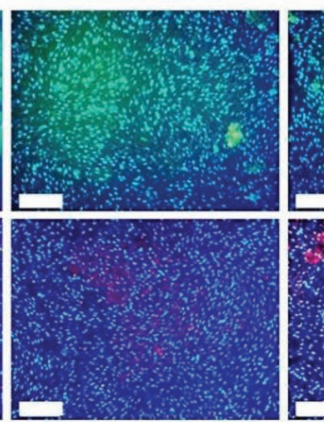

2Mo-rBGNs

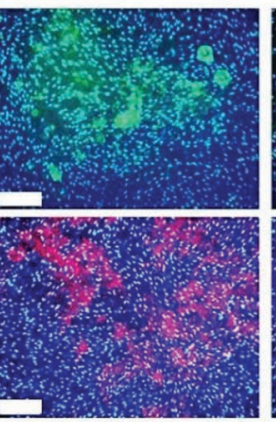

5Mo-rBGNs

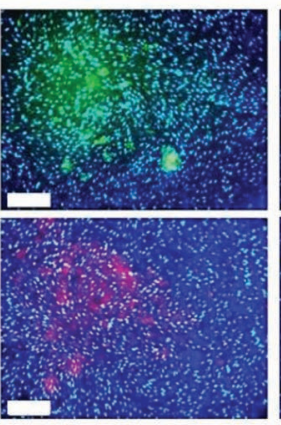

(c)

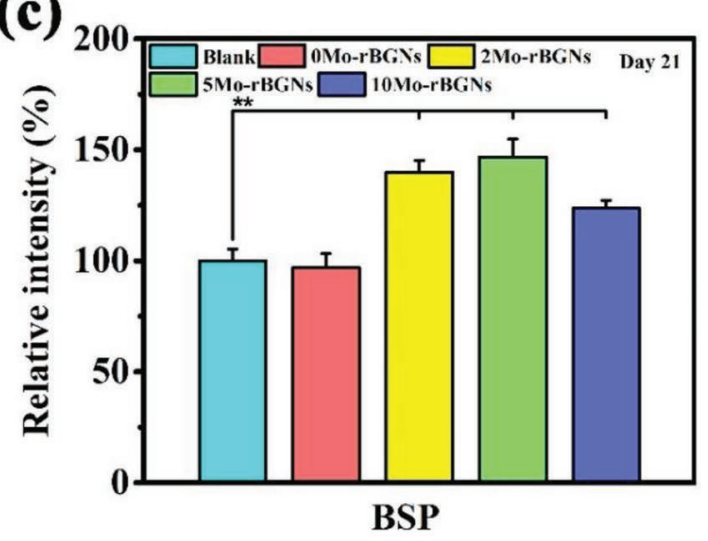

Figure 8. Immunofluorescence staining for the Col I and Bsp proteins in ADSCs on day 21 after being incubated with $60 \mu \mathrm{g} \mathrm{mL}^{-1} \mathrm{Mo-rBGNs.} \mathrm{a)} \mathrm{Immu-}$ nofluorescent staining of Col I and Bsp proteins (scale bar: $100 \mu \mathrm{m}$ ); b) relative fluorescent intensity of Col I protein ( $* p<0.05 ; * * p<0.01$ ); $\mathrm{c}$ ) relative fluorescent intensity of Bsp protein (*p $<0.05$; *** $<0.01$ ).

and 10Mo-rBGNs, accordingly. The types and sources of chemicals are shown in the Supporting Information.

Physicochemical Structure Characterizations of Mo-rBGNs: The morphology and particle size of the Mo-rBGNs were analyzed using a transmission electron microscope (JEOL JEM-F200) working at $100 \mathrm{kV}$. The phase analysis of samples was examined by X-ray diffractometer (D/MAX-RB, Rigaku) with a Ni-filtered $\mathrm{Cu} \mathrm{K} \alpha$ irradiation at $40 \mathrm{kV}$ and $30 \mathrm{~mA}$. The elemental composition of samples was determined by energy dispersive X-ray analysis (Quanta 250 FEG, FEI). The infrared spectra of the samples were obtained using a Fourier transformer infrared spectrophotometer (Nicolet 6700), in a wave number range of $400-4000 \mathrm{~cm}^{-1}$. The analyses of surface element type and atom valance were performed using X-ray photoelectron spectroscopy (Thermo Fisher ESCALAB Xi ${ }^{+}$). The surface charge of Mo-rBGNs was characterized using $\zeta$-potential measurement on a Zetasizer Nano ZS (Nano-ZS, Malvern) instrument operating with a dynamic light scattering detector positioned at $90^{\circ}$ and the test samples were suspended in $3 \mathrm{~mL}$ deionized water at a concentration of $1 \mathrm{mg} \mathrm{mL}^{-1}$.

Biomineralization Activity Assessment of Mo-rBGNs: In vitro biomineralization activity was evaluated through the methodology described in the previous report where the protocol for the assessment of hydroxyapatite forming ability of bioactive glass particles is proposed. [39] The structure and composition of formed hydroxyapatite on the surface of Mo-rBGNs was identified through XRD, FTIR, and TEM respectively. The detailed procedure is shown in the Supporting Information.

In Vitro Cellular-Biocompatibility Assessment of Mo-rBGNs: Adiposederived stem cells (Cellbank, Shanghai) were used to investigate the in vitro cellular biocompatibility of Mo-rBCNs. ADSCs were obtained from ATCC (American Type Culture Collection). The cytotoxicity and cell viability of Mo-rBGNs were studied by the alamar blue assay kit (Thermo
Fisher Scientific). The morphology and attachment of cells were also determined following LIVE/DEAD assay kit (Invitrogen). These testing procedures were according to the manufacturer's instructions. The Supporting Information shows the detailed description for cell culture, alamar blue, and LIVE/DEAD analysis.

Cellular Biomineralization and Osteogenic Differentiation Analysis: The alizarin red stain (Genmed) assay was used to indicate the results of calcium deposition and extracellular-matrix mineralization of ADSCs. After incubation with differentiation medium including Mo-rBGNs in 24-well plates at a density of $2 \times 10^{4}$ cells $\mathrm{mL}^{-1}$ for 14 and $21 \mathrm{~d}$, cells were fixed for $20 \mathrm{~min}$ using $4 \%$ paraformaldehyde, washed by PBS, and stained by $200 \mu \mathrm{L}$ alizarin for $24 \mathrm{~h}$ at room temperature. The stained cells were captured by a fluorescence microscope (IX53, Olympus). Finally, the calcium content of the differentiated ADSCs induced by Mo-rBGNs was calculated as the relative gray intensity of cells.

As an early marker of osteogenic differentiation, the ALP activities of ADSCs were evaluated on days 7, 14, and 21, using the Sensolyte pNPP ALP assay (AnaSpec), according to the manufacturer's instructions. The detailed procedure can be found in the Supporting Information. The immunofluorescence staining of osteogenic proteins (Col I and Bsp proteins) were assessed on $21 \mathrm{~d}$. The methods and procedures were similar with previous report with little change (Supporting Information). The quantitative real-time polymerase chain reaction (qRT-PCR) technique was employed to validate the expression patterns of osteogenic genes including Col I and Bsp. The primers for qRT-PCR are shown in Table S1 in the Supporting Information. And the procedures including the extraction of total RNA and analysis of qRT-PCR are indicated in the Supporting Information.

Statistical Analysis: The experiments were tested at least in triplicate and all data were expressed as the value mean \pm standard deviation 
(SD) ( $n=5$ per group). The statistical analyses were assessed by the SPSS software (version 13.0). The Student's $t$-test analysis of variance was employed to determine the significant differences between different groups. $* p<0.05$ and $* * p<0.01$ were counted statistically significant.

\section{Supporting Information}

Supporting Information is available from the Wiley Online Library or from the author.

\section{Acknowledgements}

This work was supported by the National Natural Science Foundation of China (Grant No. 51872224) and the Key Laboratory of Shaanxi Province for Craniofacial Precision Medicine Research, College of Stomatology, Xi'an Jiaotong University (Grant No. 2018LHM-KFKT004).

\section{Conflict of Interest}

The authors declare no conflict of interest.

\section{Keywords}

adipose-derived stem cells, bioactive glass nanoparticles, osteogenic differentiation, trace elements doping

Received: March 7, 2019

Revised: April 10, 2019

Published online: May 30, 2019

[1] H. Petite, U. Viateau, W. Bensaïd, A. Meunier, C. Pollak, M. Bourguignon, K. Oudina, L. Sedel, G. Guillemin, Nat. Biotechnol. 2000, 18, 959.

[2] C. Yang, X. Wang, B. Ma, H. Zhu, Z. Huan, N. Ma, C. Wu, J. Chang, ACS Appl. Mater. Interfaces 2017, 9, 5757.

[3] F. Zhao, B. Lei, X. Li, Y. Mao, R. Wang, D. Chen, X. Chen, Biomaterials 2018, 178, 36 .

[4] B. Sui, C. Hu, A. Liu, C. Zheng, K. Xuan, Y. Jin, Biomaterials 2017, 10, 046 .

[5] M. Wang, Y. Guo, M. Yu, P. X. Ma, C. Mao, B. Lei, Acta Biomater. 2017, 54, 69 .

[6] M. Gnecchi, P. Danieli, G. Malpasso, M. C. Ciuffreda, Methods Mol. Biol. 2016, 1416, 123.

[7] T. L. Tsai, W. Li, Stem Cell Rep. 2017, 8, 387.

[8] E. E. L. Lewis, H. Wheadon, N. Lewis, J. Yang, M. Mulling, A. Hursthouse, D. Stirling, M. J. Dalby, C. C. Berry, ACS Nano 2016, $10,8346$.

[9] Y. Wang, X. Chen, W. Cao, Y. Shi, Nat. Immunol. 2014, 15, 1009.

[10] W. Li, Y. Liu, P. Zhang, Y. Tang, M. Zhou, W. Jiang, X. Zhang, G. Wu, Y. Zhou, ACS Appl. Mater. Interfaces 2018, 10, 5240.

[11] R. Dai, Z. Wang, R. Samanipour, K. I. Koo, K. Kim, Stem Cells Int. 2016, 2016, 6737345.

[12] S. Zhang, B. Ma, F. Liu, J. Duan, S. Wang, J. Qiu, D. Li, Y. Sang, C. Liu, D. Liu, H. Liu, Nano Lett. 2018, 18, 2243.

[13] C. Fan, L. Jia, Y. Zheng, C. Jin, Y. Liu, H. Liu, Y. Zhou, Stem Cell Rep. 2016, 7, 236.
[14] X. Qu, Z. Liu, B. Ma, N. Li, H. Zhao, T. Yang, Y. Xue, X. Zhang, Y. Shao, Y. Chang, J. Xun, B. Lei, Y. Du, Nano Res. 2019, 12, 593.

[15] Q. Huang, Y. Zou, M. C. Arno, S. Chen, T. Wang, J. Gao, A. P. Dove, J. Du, Chem. Soc. Rev. 2017, 46, 6255.

[16] M. K. Nguyen, O. Jeon, M. D. Krebs, D. Schapira, E. Alsberg, Biomaterials 2014, 35, 6278.

[17] R. A. Marklein, J. A. Burdick, Adv. Mater. 2010, 22, 175.

[18] E. R. Balmayor, J. P. Geiger, M. K. Aneja, T. Berezhanskyy, M. Utzinger, O. Mykhaylyk, C. Rudolph, C. Plank, Biomaterials 2016, 87, 131.

[19] Y. Li, N. Li, J. Ge, Y. Xue, W. Niu, M. Chen, Y. Du, P. X. Ma, B. Lei, Biomaterials 2019, 201, 68.

[20] M. Wang, Y. Guo, Y. Xue, W. Niu, M. Chen, P. X. Ma, B. Lei, Biomaterials 2019, 199, 10.

[21] C. Wang, M. Wang, T. Xu, X. Zhang, C. Lin, W. Gao, H. Xu, B. Lei, C. Mao, Theranostics 2019, 9, 65.

[22] L. Zhou, Y. Xi, Y. Xue, M. Wang, Y. Liu, Y. Guo, B. Lei, Adv. Funct. Mater. 2019, https://doi.org/10.1002/adfm.201806883.

[23] O. Chaudhuri, L. Gu, D. Klumpers, M. Darnell, S. A. Bencherif, J. C. Weaver, N. Huebsch, H. Lee, E. Lippens, G. N. Duda, D. J. Mooney, Nat. Mater. 2016, 15, 326.

[24] M. Bohner, G. Baroud, A. Bernstein, N. Dobelin, L. Galea, B. Hesse, R. Heuberger, S. Meille, P. Michel, B. V. Rechenberg, J. Sague, H. Seeherman, Mater. Today 2017, 20, 106.

[25] T. Ting, W. Xie, W. Gao, G. Wang, L. Zeng, G. Miao, B. Lei, Z. Lin, X. Chen, Front. Chem. 2019, 7, 186.

[26] B. Lei, K. H. Shin, Y. H. Koh, H. E. Kim, J. Biomed. Mater. Res., Part B 2014, 102, 1528.

[27] J. Hum, A. R. Boccaccini, Int. J. Mol. Sci. 2018, 19, 1807.

[28] B. Lei, X. Chen, Y. Wang, N. Zhao, C. Du, L. Fang, Biomed. Mater. 2010, 5, 054103.

[29] Y. Zhou, M. Shi, J. R. Jones, Z. Chen, J. Chang, C. Wu, Y. Xiao, Int. Mater. Rev. 2017, 62, 392.

[30] Y. Xue, Z. Zhang, W. Niu, M. Chen, M. Wang, Y. Guo, C. Mao, C. Lin, B. Lei, Part. Part. Syst. Charact. 2019, 4, 1800507.

[31] L. L. Hench, J. M. Polak, Science 2002, 295, 1014.

[32] B. Lei, X. Chen, X. Han, J. Zhou, J. Mater. Chem. 2012, 22, 16906.

[33] A. El-Fiqi, T. H. Kim, M. Kim, M. Eltohamy, J. E. Won, E. J. Lee, H. W. Kim, Nanoscale 2012, 4, 7475 .

[34] W. Gao, W. Jin, Y. Li, L. Wan, C. Wang, C. Lin, X. Chen, B. Lei, C. Mao, J. Mater. Chem. B 2017, 5, 7285.

[35] J. Ge, K. Liu, W. Niu, M. Chen, M. Wang, Y. Xue, C. Gao, P. X. Ma, B. Lei, Biomaterials 2018, 175, 19.

[36] Y. Xi, J. Ge, Y. Guo, B. Lei, P. X. Ma, ACS Nano 2018, 12, 10772.

[37] A. Bachhuka, B. Delalat, S. R. Ghaemi, S. Gronthos, N. H. Voelcker, K. Vasilev, Nanoscale 2017, 9, 14248.

[38] B. Holmes, K. Bulusu, M. Plesniak, G. L. Zhang, Nanotechnology 2016, 27, 064001.

[39] Y. Xue, Y. Du, J. Yan, Z. Liu, P. X. Ma, X. Chen, B. Lei, J. Mater. Chem. B 2015, 3, 3831.

[40] M. Yu, Y. Xue, P. X. Ma, C. Mao, B. Lei, ACS Appl. Mater. Interfaces 2017, 9, 8460

[41] Y. Xue, Y. Guo, M. Yu, M. Wang, P. X. Ma, B. Lei, Adv. Healthcare Mater. 2017, 6, 1700630.

[42] Y. Li, Y. Guo, W. Niu, M. Chen, Y. Xue, J. Ge, P. X. Ma, B. Lei, ACS Appl. Mater. Interfaces 2018, 10, 17722.

[43] M. Yu, B. Lei, C. Gao, J. Yan, P. X. Ma, Nano Res. 2017, 10, 49.

[44] Y. Guo, Y. Xue, W. Niu, M. Chen, M. Wang, P. X. Ma, B. Lei, Part. Part. Syst. Charact. 2018, 35, 1800087.

[45] A. Bari, N. Bloise, S. Fiorilli, G. Novajra, M. Vallet-Regi, G. Bruni, A. Torres-Pardo, J. M. Gonzalez-Calbet, L. Visai, C. Vitale-Brovarone, Acta Biomater. 2017, 55, 493. 
[46] D. Bellucci, V. Cannillo, Mater. Lett. 2018, 213, 67.

[47] M. Wu, Y. Xue, N. Li, H. Zhao, B. Lei, M. Wang, J. Wang, M. Luo, C. Zhang, Y. Du, C. Yan, Angew. Chem., Int. Ed. 2019, https://doi. org/10.1002/ange.201812972.

[48] F. Li, M. Wang, G. Pi, B. Lei, J. Biomed. Nanotechnol. 2018, 14, 756 .
[49] E. C. Derenzo, E. Kaleita, P. G. Heytler, J. J. Oleson, B. L. Hutchings, J. H. Williams, Arch. Biochem. Biophys. 1953, 45, 247.

[50] I. A. Castro, R. S. Datta, J. Ou, A. Castellanos-Gomez, S. Sriram, T. Daeneke, K. Kalantar-Zadeh, Adv. Mater. 2017, 29, 1701619.

[51] K. Nganvongpanit, K. Buddhachat, P. Piboon, T. Euppayo, P. Mahakkanukrauh, Forensic Sci. Int. 2017, 271, 33. 